\title{
Kardiale Bildgebung mittels Computertomografie durch Kardiologen?
}

Mitglieder der Arbeitsgruppe Kardio-CT der Deutschen Gesellschaft für Kardiologie, Schmermund, Achenbach et. al., haben in Der Kardiologe 2015, S. 363-374 den Beitrag „Curriculum kardiale Computertomographie“ veröffentlicht. Ziel des Curriculums ist eine Strukturierung der Ausbildung von Kardiologen im Bereich der kardialen CT. Das Curriculum beschreibt nach den Angaben der Autoren „einen strukturierten Ausbildungsprozess, mit dem Kardiologen die Zusatzqualifikation „kardiale Computertomographie (CT)“ erlangen können." Die Ausbildung soll von der Fachgesellschaft definiert und nach einheitlichen Qualitätskriterien zertifiziert werden. Es soll die nach heutigem Stand medizinisch richtige Indikationsstellung, Anwendung und Befundinterpretation gewährleisten. Fraglich ist jedoch, ob durch das angebotene Curriculum für die Erlangung von Zusatzqualifikationen innerhalb der Kardiologie die strahlenschutzrechtlichen, weiterbildungsrechtlichen und vertragsarztrechtlichen Anforderungen erfüllt werden.

\section{Strahlenschutzrechtliche Einordnung \\ $\nabla$}

Da es sich bei der Computertomografie um eine Untersuchungsmethode mittels Röntgenstrahlen handelt, deren Anwendung den Vorschriften der Röntgenverordnung (RöV) unterfällt, stellt sich an erster Stelle die Frage, ob die Kardiologen, im Rahmen des Erwerbes der Zusatzqualifikation nach dem vorliegenden Curriculum, auch die für den Strahlenschutz erforderliche Fachkunde erhalten können.

Für die „Teilgebietsradiologie“ gilt $\S 24$ Abs. 1 Nr. 2 der Röntgenverordnung (RöV), wonach Röntgenstrahlung am Menschen nur durch Personen angewendet werden darf, die als Ärzte oder Zahnärzte approbiert sind oder denen die Ausübung des ärztlichen oder zahnärztlichen Berufs erlaubt ist und die für das Teilgebiet der Anwendung von Röntgenstrahlung, in dem sie tätig sind, die erforderliche Fachkunde im Strahlenschutz besitzen. Diese kann gemäß § 18a Abs. 1 RöV i.V.m. der Richtlinie Fachkunde und Kenntnisse im Strahlenschutz bei dem Betrieb von Röntgeneinrichtungen in der Medizin oder Zahnmedizin vom 22.12.2005 in der Fassung vom 26.06.2012 (vgl. RdSchr. d. BMU v. 27.6.2012 - RS II 4 - 11603/01, GMBl. 2012, Nr. 40, S. 724) erworben werden. Danach ist der Fachkundenachweis unter Angabe von Zeiträumen und durchgeführten Untersuchungen oder Behandlungen und die erfolgreiche Kursteilnahme durch Bescheinigungen $\mathrm{zu}$ belegen. Der Erwerb der erforderlichen Fachkunde im Strahlenschutz nach $\S 18$ a RöV wird von der zuständigen Stelle geprüft und bescheinigt.
Entsprechende Kenntnisse im Strahlenschutz sind im Regelfall kein Teil der Weiterbildung zum Facharzt/zur Fachärztin für Innere Medizin und Kardiologie und in der entsprechenden Ziffer 13.6 der Muster-Weiterbildungsordnung (MWBO) nicht enthalten. Im Rahmen der Weiterbildung zum Kardiologen ist nur die Durchleuchtung, Aufnahmetechnik und Beurteilung von Röntgenbefunden bei Angiokardiografien und Koronarangiografien Ausbildungsgegenstand. Dies bleibt weit hinter einer Teilfachkunde im Strahlenschutz zurück; insbesondere gehört die Stellung von rechtfertigenden Indikationen nach § 23 RöV nicht zum Teilgebiet der Kardiologie. Nach der Fachkunderichtlinie existiert auch kein Teilgebiet einer kardialen CT-Fachkunde. Dies erscheint auch sinnvoll, da aufgrund der hohen Strahlenbelastung in dieser Körperregion und möglichen Wechselwirkungen gerade bei der Stellung der rechtfertigenden Indikation, aber auch der Aufklärung des Patienten, eine rein singuläre kardiale CT-Fachkunde nicht ausreichend erscheint. Aus dem Curriculum ist jedoch nicht erkennbar - und wohl auch nicht beabsichtigt - dass der entsprechende Teilnehmer eine umfassende CT-Fachkunde nach Ziffer Rö5.1 der Fachkunderichtlinie erwirbt (vgl. Tabelle 4.2.1 Anforderungen zum Sachkundeerwerb für Ärzte). Das Curriculum spricht zwar davon, dass die Sachkunde im Strahlenschutz „Gefäßsystem des Herzens" Voraussetzung für die Anerkennung der Zusatzqualifikation ist. Allerdings handelt es sich bei dieser Sachkunde nach Ziffer Rö3.5 der Fachkunderichtlinie um Röntgen- und nicht um CTDiagnostik.

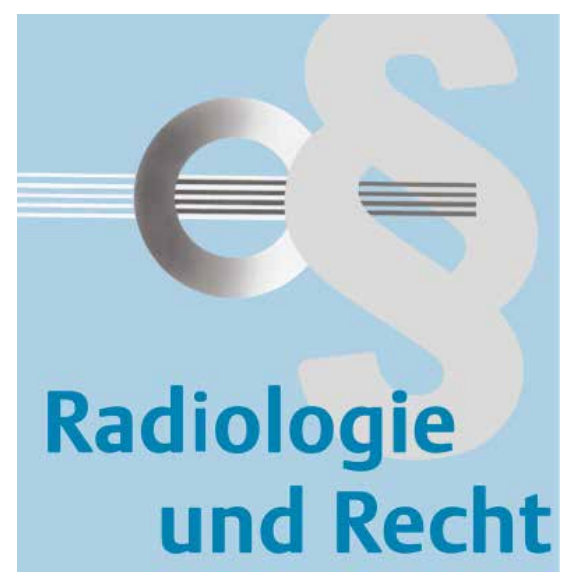

Die Erbringung von Röntgenleistungen ohne Innehaben der entsprechenden Fachkunde ist unzulässig und stellt eine Ordnungswidrigkeit gemäß $§ 44$ Nr. 12 RöV dar.

\section{Weiterbildungsrechtliche Beurteilung $\nabla$}

$\S 2$ Abs. 2 S. 2 der (Muster-)Weiterbildungsordnung (MWBO) bestimmt, dass die Gebietsdefinition die Grenzen für die Ausübung der fachärztlichen Tätigkeit bestimmt.

Dies wird flankiert von den Heilberufegesetzen der Länder. Exemplarisch schreibt $\S 41$ Abs. 1 des nordrhein-westfälischen Heilberufsgesetzes (HeilBerG NRW) vor, dass ein Arzt grundsätzlich nur auf dem Gebiet tätig werden darf, dessen Bezeichnung er führt. Das bedeutet, dass der Arzt zwar in Einzel- und Notfällen, nicht aber systematisch sein Gebiet überschreiten darf. Das Oberverwaltungsgericht Niedersachen (Urt. v. 08.07.2004, Az.: 8 LC 63/02) führt hierzu Folgendes aus:

„Eine Befugnis zur Durchführung von Schutzimpfungen lässt sich auch nicht aus einer von dem Kläger aufgrund seiner Aus- und Weiterbildung sowie seiner beruflichen Erfahrung geltend gemachten besonderen individuellen Qualifikation herleiten. Denn für die Einhaltung der Fachgebietsgrenzen und die Beurteilung der Fachfremdheit kommt es auf die Vorgaben der Weiterbildungsordnung, nicht aber auf in der Person des einzelnen Arztes liegende Gesichtspunkte des Einzelfalles an (vgl. BSG, Urteil vom 18.10.1995 - 6 RKa 52/94 - NJW 1996, 3103).

Der in $§ 36$ Abs. 2 Satz 1 HKG niedergelegte Grundsatz der Fachgebietsbeschrän- 
kung lässt allerdings Raum für Durchbrechungen. Er gilt mit den Worten des Bundesverfassungsgerichts (a.a.O., S. 168) als allgemeine Richtlinie und kann nicht als eine auch einzelne Ausnahmefälle ausschließende Regel aufgefasst werden. Den Gegensatz zu zulässigen Ausnahmefällen in diesem Sinne bildet eine unzulässige systematische Tätigkeit (vgl. etwa Rieger, a.a.O., Rn. 673). Die Abgrenzung kann berufsrechtlich nicht anhand einer bestimmten zulässigen Quote, etwa $1 \%$ der Fälle, erfolgen (vgl. Gerichtshof für Heilberufe Bremen, Urt. v. 21.2.1990 - HB - BA 1/88 -, MedR 1990, 279 ff.). Umgekehrt ist die gebietsfremde Tätigkeit aber auch nicht auf Notfälle oder vergleichbare Situationen beschränkt. Auszugehen ist vielmehr von einem Regel-Ausnahmeverhältnis. Für die Bejahung eines entsprechenden Ausnahmefalles müssen zwar keine Notstandssituationen vorliegen, aber doch besondere Umstände gegeben sein."

In der MWBO ist für das Fachgebiet der Inneren Medizin und Kardiologie die Durchführung kardialer CT-Untersuchungen, wie bereits oben dargestellt, nicht aufgeführt. Damit gehört die selbständige Durchführung kardialer CT-Untersuchungen grundsätzlich nicht zum Fachgebiet Innere Medizin und Kardiologie.

Im Ergebnis ist die Durchführungen kardialer CT-Untersuchungen nach der MWBO nicht Gegenstand der Facharztausbildung zum Kardiologen. Kardiologen sind daher für diese Behandlungsmethode prinzipiell nicht ausgebildet, sodass es keine gesetzliche Vermutung für eine Erbringung dieser Leistungen nach Facharztstandard gibt. Dass ein entsprechend ausgebildeter Kardiologe sich in der Leistungserbringung beschränkt, ist nicht zu erwarten.

Fraglich ist, ob diese Beurteilung durch diese Zusatzweiterbildung der Deutschen Gesellschaft für Kardiologie geändert werden kann. Im Curriculum selbst wird Folgendes ausgeführt:

„Für diese Curricula wurde der Begriff Zusatzqualifikation gewählt, um diese zusätzliche Qualifizierung unter dem Dach wissenschaftlichen Fachgesellschaft von der Regelweiter-bildung nach der (Muster-)Weiterbildungsordnung der Bundesärztekammer zu unterscheiden.“

Die Frage, inwieweit durch derartige Fortbildungen überhaupt Befähigungsnach- weise erbracht werden können, wird in der Literatur kritisch gesehen (Narr, Ärztliches Berufsrecht, Band 1, S. 297 ff.). Es wird dort ausgeführt, dass die Heilberufegesetze der Länder, zum Beispiel § 35 Abs. $3 \mathrm{HKaG}$ in Bayern die Möglichkeit böten, Elemente der Weiterbildung und der Fortbildung zu vermischen. Dadurch könnten Fachkunden nachgewiesen werden, ohne dass eine spezielle weiterbildungsrechtliche Form strikt eingehalten werden müsse. Ob dies jedoch hier zutrifft, dürfte fraglich sein, da als Regelungsort in $\S 35$ Abs. $3 \mathrm{HKaG}$ die Weiterbildungsordnung genannt wird. Es ist zudem aus dem vorliegenden "Curriculum“ auch nicht erkennbar, dass die Ausbildung vergleichbar intensiv abläuft wie im Rahmen der radiologischen Facharztausbildung.

Eine andere Möglichkeit für eine Rechtsgrundlage, die in der Literatur erwogen wird, könnte die allgemeine ärztliche Fortbildungspflicht (z. B. § $18 \mathrm{HKaG}$ ) sein (Narr, a. a. O.). Allerdings dürfte diese Vorschrift viel zu unbestimmt sein, um eine entsprechende Ermächtigungsgrundlage darzustellen. Daher dürften die entsprechenden Qualifikationen keinen „offiziellen" berufsrechtlichen Charakter haben. Eine Erbringung kardialer CT-Untersuchungen von Kardiologen stellt daher trotz der Absolvierung des Curriculums unseres Erachtens eine systematische Überschreitung der Fachgebietsgrenzen dar, die berufsrechtliche Sanktionen durch die Landesärztekammern zur Folge haben kann. Dies gilt insbesondere dann, wenn der Arzt erkennt, dass er sein Fachgebiet überschreitet (vgl. Gerichtshof für die Heilberufe beim OVG Bremen, Urt. v. 21.02.1990, Az.: OVG HB-BA 1/88).

Es kann festgehalten werden, dass eine selbständige Durchführung und Befundung kardialer CT-Leistungen weiterbildungsrechtlich trotz des Curriculums ausschließlich Radiologen vorbehalten bleibt. Für Kardiologen ist die Erbringung dieser Leistungen daher fachgebiets-fremd und damit berufsrechtlich unzulässig. Die selbstständige Erbringung dieser Leistungen ist folglich Kardiologen prinzipiell untersagt.

Im Ergebnis wird die Computertomografie in der MWBO und den Weiterbildungsordnungen der Ärztekammern dem Gebiet der Radiologie als „Definiertes Untersuchungs- und Behandlungsverfahren“ zugewiesen. Eine derartige Zuweisung findet sich für kein anderes Fachgebiet im
Weiterbildungsrecht. Der Facharzt für Strahlentherapie ist z.B. zwar zur Durchführung von CT-Untersuchungen berechtigt, jedoch beschränkt sich diese auf die „Therapieplanung“ in seinem Fachgebiet (vgl. Wigge, Kaiser, Fischer, Loose, MedR 2014, 700, 710). Eine eigenständige Diagnostik ist ihm daher verwehrt. Damit gehört die selbständige Durchführung kardialer CT-Untersuchungen ausschließlich zum Fachgebiet der Radiologie, nicht dagegen zum Fachgebiet Innere Medizin und Kardiologie.

\section{Vertragsarztrechtliche Vorgaben \\ $\nabla$}

Die Anforderungen an die Abrechnung computertomografischer Leistungen in der GKV sind in der „Vereinbarung zur Strahlendiagnostik und -therapie" geregelt, die von den Vertragspartnern der Bundesmantelverträge nach $\S 135$ Abs. 2 SGB V erlassen worden ist.

Nach $\S 7$ Abs. 1 der Vereinbarung gilt die fachliche Qualifikation durch die Vorlage ausreichender Zeugnisse als nachgewiesen, soweit die Weiterbildungsordnung für eine Weiterbildung in der Computertomografie den Erwerb eingehender Kenntnisse, Erfahrungen und Fertigkeiten im jeweiligen Bereich (Ganzkörper, Kopf) vorschreibt. Diese spezifischen Anforderungen werden gegenwärtig ausschließlich in den Weiterbildungsinhalten des Fachgebietes Radiologie aufgeführt (Wigge, Fischer, Kaiser, Loose, a. a. O, S. 710).

Anderen Ärzten ist jedoch die Erbringung computertomografischer Leistungen in der GKV unter den Voraussetzungen des $\S$ 7 Abs. 2 und 3 der Vereinbarung prinzipiell möglich. Dies gilt insbesondere für Ärzte anderer Fachrichtungen, die auf der Grundlage früherer Weiterbildungsordnungen die vorgegebenen Voraussetzungen erfüllen. Soweit keine Weiterbildung nach Abs. 1 stattgefunden hat, kann ein Arzt nach Abs. 2 durch die Vorlage ausreichender Zeugnisse nachweisen, dass er während der genannten Zeiten für die Bereiche Ganzkörper und Kopf unter der Leitung eines zur Weiterbildung ermächtigten (befugten) Arztes tätig gewesen ist:

- „a Für Untersuchungen des Ganzkörpers auch einschl. Kopf und des Spinalkanals

1. eine mindestens 30monatige ganztägige Tätigkeit in der radiologischen einschl. neuroradiologischen Diagnostik und 
2. eine mindestens 10 monatige ganztägige Tätigkeit in der Computertomographie

- b für Untersuchungen des Kopfes und des Spinalkanals

1. eine mindestens 18 monatige ganztägige Tätigkeit in der radiologischen einschl. neuroradiologischen Diagnostik und

2. eine mindestens 4 monatige ganztägige Tätigkeit in der Computertomographie insbesondere des Kopfes und des Spinalkanals.“

Ärzte, die ihre fachliche Qualifikation nach $\S 7$ Abs. 2 der Vereinbarung erworben haben, müssen diese nach Abs. 3 in einem Kolloquium nach $\S 17$ Abs. 1 S. 2 der Vereinbarung nachweisen.

In $\S 7$ Abs. 2 i.V.m. $§ 17$ Abs. 1 S. 2 der Vereinbarung ist geregelt, dass für den Fall, dass der antragstellende Arzt im Vergleich zu $\S 7$ Abs. 1 der Vereinbarung eine abweichende, aber gleichwertige Befähigung im Bereich der Computertomografie nachweist, die Genehmigung für die Ausführung und Abrechnung der computertomografischen Leistungen von der erfolgreichen Teilnahme an einem Kolloquium abhängig ist:

„(1) Bestehen trotz der Berechtigung zum Führen einer Gebiets- bzw. Teilgebietsbezeichnung oder der vorgelegten Zeugnisse begründete Zweifel, dass die in Abschnitt B dieser Vereinbarung festgelegten Anforderungen an die fachlichen Befähigungen erfüllt sind, so kann die Kassenärztliche Vereinigung die Erteilung der Genehmigung für die Ausführung und Abrechnung der beantragten Leistungen von der erfolgreichen Teilnahme an einem Kolloquium abhängig machen. Das gleiche gilt, wenn der antragstellende Arzt im Vergleich zu dieser Vereinbarung eine abweichende, aber gleichwertige Befähigung nachweist.“

Auslegungsschwierigkeiten bereitet in der Praxis die Regelung in $\S 17$ Abs. 1 Satz 2 der Vereinbarung, wonach die KV die Abrechnungsgenehmigung auch dann von der erfolgreichen Teilnahme an einem Kolloquium abhängig machen kann, wenn „der antragstellende Arzt im Vergleich zu dieser Vereinbarung eine abweichende, aber gleichwertige Befähigung nachweist“.

Der in $\S 17$ Abs. 1 Satz 2 genannte Begriff der „gleichwertigen Befähigung“ bezieht sich für den Bereich der Computertomografie inhaltlich ausschließlich auf die Regelung in $\S 7$ Abs. 2 der Vereinbarung. Nach $§ 7$ Abs. 1 gilt die fachliche Qualifikation grundsätzlich durch die Vorlage ausreichender Zeugnisse als nachgewiesen, „soweit die Weiterbildungsordnung für eine Weiterbildung in der Computertomographie den Erwerb eingehender Kenntnisse, Erfahrungen und Fertigkeiten in der jeweiligen computertomographischen Diagnostik (Ganzkörper, Kopf) vorschreibt." Nur soweit dagegen eine Weiterbildung nach $\S 7$ Abs. 1 nicht stattgefunden hat, muss der Antragsteller nach Abs. 2 durch die Vorlage ausreichender Zeugnisse nachweisen, dass er während der genannten Zeiten unter der Leitung eines zur Weiterbildung ermächtigten Arztes tätig gewesen ist und daher über eine zum Fachgebiet der Radiologie „gleichwertige Befähigung“ verfügt. $§ 7$ Abs. 3 S. 1 der Vereinbarung schreibt deswegen auch vor, dass Ärzte, die ihre fachliche Qualifikation nach Abs. 2 erworben haben, diese gemäß $\S 17$ Abs. 2 in einem Kolloquium nachweisen müssen.

Abweichende, aber gleichwertige Befähigungen beziehen sich somit nach $\S 7$ Abs. 2 auf die nachzuweisende Anzahl der durchgeführten Untersuchungen des Ganzkörpers einschließlich des Kopfs und des Spinalkanals, für solche ärztlichen Fachgebiete, die anders als Fachärzte für Radiologie, nicht bereits aufgrund der Anforderungen in der Weiterbildungsordnung gemäß $§ 7$ Abs. 1 den Nachweis der fachlichen Qualifikation durch die Ableistung ihrer Weiterbildungszeit erbracht haben. Dieser Nachweis ist von allen anderen ärztlichen Fachgebieten zu erbringen, da nur das Fachgebiet der Radiologe über die nach $\S 7$ Abs. 1 geforderte „Weiterbildung in der Computertomographie“ nach den Weiterbildungsordnungen der Landesärztekammern verfügt, wonach „der Erwerb eingehender Kenntnisse, Erfahrungen und Fertigkeiten in der jeweiligen computertomographischen Diagnostik (Ganzkörper, Kopf)“ nachgewiesen werden muss.

Das vorliegende Curriculum in seiner Beschränkung auf kardiale CT umfasst allerdings weder den Bereich „Kopf“, noch den Bereich „Ganzkörper“. Zusätzlich sind jedoch auch nach der Qualitätssicherungsvereinbarung zur Strahlendiagnostik und -therapie die strahlenschutzrechtlichen Anforderungen von den Antragstellern zu erfüllen. § 4 der Vereinbarung schreibt diesbezüglich folgendes vor:

\section{„§ 4 Diagnostische Radiologie}

Den Anforderungen an die fachliche Befähigung für die Ausführung und Abrechnung von Leistungen der diagnostischen Radiologie wird entsprochen, wenn der Arzt

1. die für den Strahlenschutz erforderliche Fachkunde nach $\S 3$ Abs. 3 Nr. 2

RöV nachweist, sofern er nicht auf Grund der Übergangsvorschriften der Röntgenverordnung ( $§ 45$ Abs. 2 Satz 1 und $\S 45$ a) von der Nachweispflicht befreit ist und

2. eine fachliche Qualifikation gemäß den in den $\S \S 5$ bis 8 genannten Anforderungen erworben hat.“

Unabhängig davon, dass CT-Untersuchungen nach dem Weiterbildungsrecht nicht zum Fachgebiet Innere Medizin und Kardiologie gehören, dürften die Voraussetzungen für das Genehmigungsverfahren für diese radiologischen Leistungen nach der Qualitätssicherungsvereinbarung zur Strahlendiagnostik und -therapie nicht gegeben sein, weil Kardiologen nicht über die erforderliche Fachkunde im Strahlenschutz nach § 4 der Vereinbarung i.V.m. § 18a RöV und der Richtlinie Fachkunde und Kenntnisse im Strahlenschutz bei dem Betrieb von Röntgeneinrichtungen in der Medizin oder Zahnmedizin vom 22.12.2005 in der Fassung vom 26.06.2012 verfügen.

Schließlich ist allerdings auch aufgrund der Regelung in $§ 135$ Abs. 2 Satz 4 SGB V davon auszugehen, dass die Erbringung computertomografischer Leistungen in der vertragsärztlichen Versorgung ausschließlich Fachärzten für Radiologie und Fachärzten vorbehalten ist, die auf der Grundlage älterer Weiterbildungsordnungen auch eine Fachkunde im Strahlenschutz erhalten haben. Aufgrund der aktuell geltenden Anforderungen nach den Weiterbildungsordnungen werden die Anforderungen jedoch ausschließlich von Fachärzten für Radiologie erfüllt. Die Entscheidungen des BVerfG und des BSG zur Kernspintomografie-Vereinbarung belegen, dass die gesetzliche Regelung in $§ 135$ Abs. 2 Satz 4 SGB V die Beschränkung der Erbringung und Abrechnung von computertomografischen Leistungen auf das Fachgebiet der Radiologie zulässt. 
Das Bundesverfassungsgericht hat bereits in seiner Entscheidung v. 16.7.2004 (Az.: 1 BvR 1127/01 -, MedR 2004, 608 ff.) festgestellt, dass der Ausschluss von der Abrechenbarkeit kernspintomografischer Leistungen in der vertragsärztlichen Versorgung auch für Orthopäden einen verfassungsrechtlich gerechtfertigten Eingriff in die Berufsfreiheit nach Art. 12 Abs. 1 GG darstellt. Das BSG hatte sich in seinem Urteil v. 11.10.2006 (Az.: B 6 KA 1/05 R -, GesR 2007, 209 ff.) auf die vorhergehende Entscheidung des BVerfG gestützt, wonach „die Konzentration aller kernspintomografischen Leistungen bei speziell qualifizierten Ärzten“ zur Sicherung der Wirtschaftlichkeit der vertragsärztlichen Versorgung zulässig sei. Weshalb diese Feststellung nicht zumindest grundsätzlich auch für Kardiologen gelten sollte, war für das BVerfG nicht ersichtlich (vgl. BVerfG, Beschl. v. 8. 7. 2010, Az.: 2 BvR 520/07). Hinzu kommt nach Ansicht des BVerfG, dass durch das Gesetz zur Modernisierung der Gesetzlichen Krankenversicherung (GKV-Modernisierungsgesetz (GMG) v. 14.11.2003, BGBl. I 2190) § 135 Abs. 2 SGB V zum 1.1.2004 um einen Satz 4 ergänzt worden ist. Dort wird folgendes bestimmt:

„Abweichend von Satz 2 können die Vertragspartner nach Satz 1 zur Sicherung der Qualität und Wirtschaftlichkeit der Leistungserbringung Regelungen treffen, nach denen die Erbringung bestimmter medizinisch-technischer Leistungen den Fachärzten vorbehalten ist, für die diese Leistungen zum Kern ihres Fachgebietes gehören.“

In der Begründung der Fraktionen der SPD, CDU/CSU und Bündnis 90/Die Grünen zu dieser Ergänzung des $\S 135$ Abs. 2 SGB V wurde ausdrücklich auf das BSGUrteil vom 31.1.2001 (Az.: B 6 KA 24/00 R -) Bezug genommen und die Notwendigkeit betont, die Durchführung diagnostischer Maßnahmen (medizinisch-technischer Leistungen) auch dann bei den dafür spezialisierten Ärzten zu konzentrieren, wenn diese Leistungen nach dem landesrechtlichen Berufsrecht (auch) zum Fachgebiet des "therapeutisch tätigen Arztes“ zählen (BT-Dr. 15/1525 S. 124). Deshalb sind spätestens nach Inkrafttreten des GMG die Überlegungen therapeutisch tätiger Fachgruppen, inwieweit sich aus den aktuellen Änderungen im ärztlichen Weiterbildungsrecht Gesichtspunkte für die Zugehörigkeit kernspintomografischer Diagnostik auch zum jeweiligen Fachge- biet (Chirurgie, Orthopädie, Innere Medizin, Gynäkologie) ergeben können, für die hier allein betroffene vertragsärztliche Versorgung ohne Bedeutung.

Wie das BSG (BSG, Urt. v. 11.10.2006 Az. B 6 KA 1/05 R -, a.a.O. S. 211) ausführt, sei im Einzelfall nie auszuschließen, dass ein Arzt einer bestimmten Fachrichtung für eine bestimmte hochspezialisierte Leistung in besonderer Weise qualifiziert ist, die üblicherweise von Ärzten einer anderen Fachrichtung erbracht wird, und dass umgekehrt ein Facharzt im Rahmen seiner Weiterbildung mit einer ganz speziellen Leistung nur am Rande befasst worden ist. An derartig untypischen Situationen müssten sich die Normgeber weder auf der Ebene des Gesetzes, noch im Rahmen von Qualitätssicherungsvereinbarungen auf der Grundlage des $\S 135$ Abs. 2 SGB V orientieren. Normsetzung dürfe von typischen Sachverhalten und Konstellationen ausgehen und einem typischen Sachverhalt entspreche es, dass Ärzte, die langjährige Tätigkeit und Erfahrung in der Kernspintomografie hätten, die erforderliche Qualifikation zur Durchführung zumindest derjenigen kernspintomografischen Untersuchungen der Herzregion besäßen, die derzeit bereits Gegenstand der vertragsärztlichen Versorgung seien.

Diese Ausführungen des BSG sind aufgrund der vergleichbaren Genehmigungskonstellation in $\S 7$ Abs. 1 und 2 der Qualitätssicherungsvereinbarung zur Strahlendiagnostik und -therapie auf das dort geregelte Genehmigungsverfahren für computertomografische Leistungen übertragbar. Die Erteilung einer Genehmigung zur Durchführung und Abrechnung von Leistungen der Computertomografie für das Fachgebiet Innere Medizin und Kardiologie im Rahmen der vertragsärztlichen Versorgung auf der Grundlage der Regelung in $\S 7$ Abs. 2 und 3 i.V.m. $§ 17$ der Qualitätssicherungsvereinbarung wäre daher rechtswidrig, weil dadurch gegen $\S$ 135 Abs. 2 Satz 4 SGB V verstoßen würde.

Die Tätigkeit der Kardiologen bei kardialen CT-Untersuchungen dürfte zudem unter diesem Gesichtspunkt auch gegen das Verbot der Erbringung fachgebietsfremder Leistungen im vertragsärztlichen Bereich verstoßen. Das BSG hat in seiner Rechtsprechung dabei objektive und subjektive Kriterien aufgestellt. Aus subjektiver Sicht muss der Behandlungsanlass stets auf Leistungen für fachgebietseigene Indikationen bezogen sein (BSG, Urt. v.
05.02.2003, Az.: B 6 KA 15/02 R). Dies mag man vorliegend noch annehmen, denn die Untersuchungen sind ja im Fachgebiet der Kardiologie begründet. Problematischer ist jedoch die objektive Beurteilung. Danach liegt die Grenze einer gebietsärztlichen Betätigung dann vor, wenn Leistungen „nach Methode oder nach Körperregion nur von anderen Gebietsärzten erbracht werden dürfen." (BSG, a. a. O.). Weiterbildungs- und strahlenschutzrechtlich kann die entsprechende Leistung, wie oben gezeigt, nur durch Fachärzte für Radiologie bzw. durch Ärzte mit der entsprechenden Fachkunde im Strahlenschutz erbracht werden.

Schließlich sei darauf hingewiesen, dass im Rahmen der Vereinbarung zur Strahlendiagnostik und -therapie keine Einzelqualifikation für kardiale Computertomografie geregelt ist. § 7 dieser Vereinbarung differenziert nur zwischen Qualifikationen für die Bereiche Ganzkörper bzw. Kopf und Spinalkanal. Durch das vorgeschlagene „Curriculum kardiale Computertomographie“ werden diese Anforderungen nicht erfüllt

\section{Arzthaftungsrechtliche Konsequenzen \\ $\nabla$}

Die fehlende Zugehörigkeit einer ärztlichen Maßnahme zum Fachgebiet eines Arztes kann zugleich einen ärztlichen Behandlungsfehler implizieren, falls dem Patienten aufgrund der Tätigkeit ein Gesundheitsschaden erwächst, da eine fachgebietsfremde Leistung grundsätzlich nicht dem ärztlichen Standard entspricht. Dieser Facharztstandard ist durch die Regelung in $\S 630$ a Abs. 2 BGB auch ausdrücklich gesetzlich vorgeschrieben.

Der Facharztstandard ist bei der haftungsrechtlichen Beurteilung nach der Rechtsprechung des BGH entscheidend (BGH, Urt. v. 21.11.1995, Az.: VI ZR 341/94). Dies gilt auch in dem Fall, in dem der entsprechende Arzt zwar Facharzt, aber nicht des entsprechenden Fachgebiets ist (BGH, Urt. v. 27.10.1981, Az.: VI ZR 69/80). Bei einer kardialen CT-Untersuchung muss der Kardiologe somit vollständig den Facharztstandard eines Radiologen beachten. Führt er eine Behandlung durch, ohne die entsprechenden Fähigkeiten und Kenntnisse zu haben, ist darin ein Behandlungsfehler zu sehen. Hier ist insbesondere daran zu denken, dass der Kardiologe ausweislich des Curriculums keine Kenntnis- 
se der CT-Diagnostik der benachbarten Organsysteme erwirbt, was auch für Stellung einer rechtfertigenden Indikation wichtig ist. Zudem ist dies ggf. auch Teil der Aufklärung des Patienten, die durch den Kardiologen so nicht sachgerecht durchgeführt werden kann. Diese rechtliche Bewertung hat sich durch das Patientenrechtegesetz noch weiter verschärft. Nach $\S 630 h$ BGB besteht bei mangelnder Befähigung eines Behandelnden eine gesetzliche Vermutung dafür, dass die mangelnde Befähigung für den Eintritt der Verletzung des Lebens, des Körpers oder der Gesundheit ursächlich war.

Die konkrete Konsequenz daraus ist, dass bei einem Behandlungsfehler der fachlich nicht befähigte Arzt beweisen muss, dass die Verletzung des Patienten aus einem anderen Grund eingetreten ist. Ähnlich wie bei der Beweislastumkehr bei der Haftung für grobe Behandlungsfehler $(\S 630 \mathrm{~h}$ Abs. 5 BGB) wird diese Vermutung in einem gerichtlichen Verfahren nicht zu entkräften sein.

\section{Gebührenrechtliche Konsequenzen $\nabla$}

Die Nichteinhaltung der Fachgebietsgrenzen kann auch gebührenrechtliche Konsequenzen haben. $\S 4$ Abs. 2 GOÄ bestimmt, dass der Arzt nur für selbstständige ärztliche Leistungen Gebühren abrechnen kann, die er selbst erbracht hat oder die unter seiner Aufsicht nach fachlicher Weisung erbracht wurden. Das Kriterium der fachlichen Weisung setzt voraus, dass der abrechende Arzt selbst die entsprechenden Kenntnisse hat, da der Patient das entsprechende Vertrauen in die fachliche Qualifikation des entsprechenden Arzts setzt (Brück u. a., Kommentar zur Gebührenordnung für Ärzte, § 4 GOÄ, Rn. 9). Privatärztliche Abrechnungen, die nicht den Vorgaben des $\S 4$ Abs. 2 GOÄ entsprechen, sind falsch und können nicht nur zur Rückforderung des Honorars führen, sondern auch als Abrechnungsbetrug gewertet werden.

\section{Ergebnis \\ $\nabla$}

Im Ergebnis ist festzuhalten, dass das Curriculum kardiale CT der Deutschen Gesellschaft für Kardiologie den teilnehmenden
Kardiologen, insbesondere aus strahlenschutz- und weiterbildungsrechtlichen, aber auch aus anderen juristischen Gesichtspunkten, nicht die Möglichkeit verschafft, die fachlichen Voraussetzungen für die Erbringung und Abrechnung von computertomografischen Leistungen $\mathrm{zu}$ erwerben.

Die gegenwärtige Rechtslage passt sich auch in das gesamte System der diagnostischen Bildgebung ein, da auf diese Art und Weise dem Problem der Selbstzuweisung - gerade bei Untersuchungen, die mit einer erhöhten Strahlenexposition des betroffenen Patienten einhergehen und deshalb besonderen Anforderungen nach der RöV unterliegen - wirksam begegnet wird. Für den Bereich der vertragsärztlichen Versorgung ist an dieser Stelle anzumerken, dass den Radiologen ebenfalls nicht gestattet ist, diagnostische Leistungen zu erbringen, die zum Kernbereich des kardiologischen Fachgebiets gehören. Hierzu zählen insbesondere Linksherzkatheteruntersuchungen und therapeutische Katheterinterventionen, die nach $\S 4$ Abs. 1 Vereinbarung zur invasiven Kardiologie im Regelfall ausschließlich Kardiologen vorbehalten sind.

\section{Prof. Dr. Peter Wigge \\ Rechtsanwalt, Fachanwalt für Medizin- recht}

\section{Lic. iur. can. Urs Fabian Frigger Rechtsanwalt}

Rechtsanwälte Wigge

Scharnhorststr. 40

48151 Münster

Telefon: (0251) 53 595-0

Telefax: (0251) 53 595-99

E-Mail: kanzlei@ra-wigge.de 